https://dx.doi.org/10.4314/jpb.v17i2.2

Vol. 17 no. 2, pp. 88-95 (September 2020)

http://ajol.info/index.php/jpb

\section{Journal of \\ PHARMACY AND BIORESOURCES}

\title{
Inhibition of RNA-dependent RNA polymerase from SARS- CoV-2 by compounds in Vangag herbal preparation: an in silico evaluation
}

\author{
Kakjing D. FALANG ${ }^{1 *}$, Catherine O. POYI ${ }^{2}$ and Jacob A. KOLAWOLE ${ }^{2}$ \\ ${ }^{I}$ Department of Pharmacology; ${ }^{2}$ Department of Pharmaceutical and Medicinal Chemistry; University of Jos, \\ Jos. Nigeria.
}

Received $15^{\text {th }}$ August 2020; Accepted $3^{\text {rd }}$ September 2020

\begin{abstract}
Coronavirus disease (COVID-19) has presented unprecedented challenges to healthcare systems worldwide. There are no proven effective therapeutic agents or vaccines. Some antiviral agents and micronutrients have been repurposed for the management. There are claims of herbal preparations with therapeutic effects. Vangag herbal formulation for the management of COVID-19 is a combination of six plants. Molecular docking and virtual screening were used for the study. Ligands and protein target for molecular docking were prepared in Autodock Tools using PyRx 0.8 package. 3D structures of 24 phytochemicals in Vangag were downloaded from PubChem and optimized in Discovery Studio 4.5 visualizer. Nine agents currently used for management of COVID-19 were also downloaded and included in the ligand library to serve as control. Results of the binding affinities of phytochemicals in constituent plants of Vangag to SARS-CoV-2 molecular target (7BV2.pdb) were ranked from 1 to 21. Kolaviron (binding affinity $-8.1 \mathrm{Kcal} / \mathrm{mol}$ ) ranked 1, Ritonavir 5, Remdesivir 6, Quinine 7, Hydroxychloroquine 9, Chloroquine 18 and the least Allicin 21 (binding affinity $-3.7 \mathrm{Kcal} / \mathrm{mol}$ ). Phytochemicals in Vangag have good binding affinity to COVID-19 viral target proteins. Vangag also contains high concentration of zinc and other micronutrients, making it a promising formula for management of COVID-19.
\end{abstract}

Keywords: COVID-19; SARS-CoV-2; Molecular docking; Herbal formulation

\section{INTRODUCTION}

The use of herbs as medicines dates far back to the origin of civilization with the earliest known records in China and India $[1,2]$. The use and popularity of herbal medicinal preparations as well as supplements from same has tremendously increased in today's world. It is estimated that close to $80 \%$ of peoples across the world rely on these preparations at least for some part of primary healthcare [3]. This extensive use and the search for even more herbal remedies has somewhat accelerated exponentially in the era of the global coronavirus pandemic.

Presently, Severe Acute Respiratory Syndrome Coronavirus 2 (SARS-CoV-2 which causes Coronavirus Disease 2019 (COVID-19) has rapidly spread across the

*Correspondence. E-mail: falangkakjing@yahoo.com Tel: +234-8033930674.

ISSN 0189-8442

2020. Published by Faculty of Pharmaceutical Sciences, University of Jos, Nigeria. Under Creative Commons Attribution-NonCommercial 4.0 International License. https://creativecommons.org/licenses/by-nc/4.0/ 
world. There are no specific treatments (whether antiviral drugs or vaccines), the management consist of only supportive care and non-specific treatment. Current clinical management includes infection prevention, control measures, supportive care and the use of re-purposed drug therapy based on their chemistry, pharmacology, molecular biology and pathophysiology of the COVID-19 disease. These therapeutic agents include (but not limited to) antiviral agents (remdesivir, hydroxychloroquine, chloroquine, lopinavir, umifenovir, favipiravir, and oseltamivir), antioxidants (vitamin C) and micronutrients (zinc and selenium) [4]. It is estimated that more than $85 \%$ patients with SARS-CoV-2 have received some form of herbal treatments in china [5]. Thus, herbal medicinal preparations have been proposed as a resource that is very important for drug discovery against SARS-CoV-2 [6]. In this regard, there are literature reports on the utility of in silico techniques in the identification of herbs that may be useful in the management of viral respiratory infections and also contain compounds that might directly inhibit SARSCoV-2 [7].

In silico techniques use computational approaches to capture, analyse and integrate biological and medical data from many diverse sources. It creates computational models or simulations which may be useful in making predictions, suggestion of hypotheses and provision of discoveries and/or advances in medicine and therapeutics [8]. The herbal preparation Vangag consists of Aframomum melegueta, Gongronema latifolium, Allium sativum, Viscum album, Garcinia kola and Nigella sativa. There is no report in the literature, to the effect that this combination of plants and in the proportions employed, has ever been documented for use in the management of COVID-19. The study used in silico techniques to evaluate the phytochemical constituents present in five Nigerian medicinal plants used in the preparation of Vangag as potential inhibitors

of SARS-CoV-2 Protease.

The crystal structure of Viral protein SARS-CoV-2 protease (7BV2. pdb) used in this study was expressed in Spodoptera frugiperda and the experimental data snapshot taken at a resolution of 2.50 Angstrom. This complex is suitable for our study seeing as RNA-dependent RNA polymerase (RdRp) replication of the virus is a good potential target for ligands.

\section{EXPERIMENTAL}

Protein and ligand library preparation. Three dimensional structures of twenty-four (24) phytochemical constituents (Table 1) [9-15], contained in Vangag were downloaded from PubChem [16] and optimized in Discovery Studio 4.5 visualizer [17]. Nine therapeutic compounds currently in use for the treatment of COVID-19 [4] were also downloaded and included in the ligand library to serve as control. The downloaded therapeutic compounds included Lopinavir, Remdesivir, Ritonavir, Umifenovir, Favipiravir, Oseltamivir, Quinine, Chloroquine and Hydroxychloroquine. The crystal structure of the SARS- CoV-2 target (7BV2.pdb) was downloaded from the Protein data bank (https://www.rcsb.org/). Its original ligands (remdesivir) and water were eliminated using discovery studio 4.5 visualizer [17].

Virtual screening. Ligands and Protein target for molecular docking were prepared in Autodock Tools using PyRx 0.8 package [18]. A grid box (x: 89.4261, y: 86.5813, z: 98.8772), dimensions (Angstrom) (x: 44.1528 y: $48.0231 \mathrm{z}: 25.0000)$ was employed and docking simulations of bioactive conformations was done using Autodock Vina [19]. The ligands were inputted as Standard Database Files (.sdf files) while the target protein was inputted as a Protein Database File (.pdb file) then converted to the acceptable Protein Data Bank, Partial Charge (Q), \& Atom Type (T) format (.pdbqt file format) for 
Autodock Vina. A total of nine exhaustive conformation searches were done and the one with the lowest binding energy retained for analysis. Results obtained were analysed using PyMol [20] and Discovery Studio 4.5 visualizer [17].

\section{RESULTS}

Virtual screening. Analysed virtual screening results of the binding affinities (Kcal/mol) of phytochemical compounds contained in the plant constituents of Vangag to SARS-CoV-2 molecular target (7BV2.pdb) were ranked and are presented in Table 2.

Binding interactions. All the small molecules used in this study were observed to have docked properly in the active site of the target. Upon binding of small molecules to 7BV2.pdb, no global changes were observed. Figure 1 shows the binding interactions of remdesivir and key phytochemical compounds in the herbal preparation with to the active site residues of SARSCoV-2. Figure 2 shows the three-dimensional view of binding conformations of compounds to the active site of the enzyme. Lys 545 and Arg 555 are of interest in the finger subdomain of the nonstructural protein 12 (nsp12) RdRp domain (Residues 366- 920) of 7BV2.pdb of which most of the bioactive compounds were observed to have interacted with. Of importance is the interaction of Kolaviron with Asp 760 and Asp 761 residues which are in the catalytic center of the protein target.

Predicting binding affinity from docking scores. The energy released through formation of bond between the ligand and protein is termed the binding energy which is usually negative. A lower binding energy most times translates to a higher affinity for the target. Binding pattern analysis (Figure 1), of the compounds showed numerous hydrogen- bond and van der Waals interactions.

Table 1: The medicinal plants and their Bioactive compounds used in Docking Studies

\begin{tabular}{|c|c|c|}
\hline MEDICINAL PLANT & Bioactive Compounds & References \\
\hline \multirow{4}{*}{ Aframomum melegueta (Alligator Pepper) } & Oleanolic acid & [9] \\
\hline & Gingerdione & \\
\hline & 6- gingerol & \\
\hline & 6- paradol & \\
\hline Allum sativum (Garlic) & Allicin & {$[10,11]$} \\
\hline \multirow{9}{*}{ Viscum album (Mistletoe) } & Syringin & [12] \\
\hline & Myricetin & \\
\hline & Kaempferol & \\
\hline & Chlorogenic acid & \\
\hline & Protocatechuic acid & \\
\hline & Quercetin & \\
\hline & Rosmarinic acid & \\
\hline & Ferulic acid & \\
\hline & Sinapic acid & \\
\hline Garcinia kola (Bitter kola) & Kolaviron & [13] \\
\hline \multirow{9}{*}{ Nigella sativa (Black seed) } & Thymoquinone & {$[14,15]$} \\
\hline & Thymohydroquinone & \\
\hline & Dithymoquinone & \\
\hline & Thymol & \\
\hline & $\alpha$-pinene & \\
\hline & $\beta$-pinene & \\
\hline & d-limonene & \\
\hline & p- cymene & \\
\hline & Citronellal & \\
\hline
\end{tabular}


Table 2: Binding Affinities of Bioactive Compounds to SARS-CoV-2 Molecular Target (7BV2.pdb)

\begin{tabular}{|c|c|c|c|}
\hline Phytochemical constituents & Binding Affinity (Kcal/mol) & Rank & Type of interactions \\
\hline Kolaviron & -8.1 & 1 & Van der Waals, hydrogen bonds \\
\hline Quercetin & -7.9 & 2 & $\begin{array}{l}\text { Van der Waals, hydrogen bonds, Unfavourable } \\
\text { donor-donor, carbon hydrogen bond interactions }\end{array}$ \\
\hline Kaempferol & -7.9 & 2 & Van der Waals, hydrogen bonds \\
\hline Myricetin & -7.9 & 2 & Van der Waals, hydrogen bonds \\
\hline Chlorogenic acid & -7.4 & 3 & $\begin{array}{l}\text { Van der Waals, hydrogen bonds, carbon } \\
\text { hydrogen bonds, Unfavourable Acceptor- } \\
\text { Acceptor, Pi-Anion and Pi-alkyl interactions }\end{array}$ \\
\hline Rosmarinic acid & -7.2 & 4 & Van der Waals, hydrogen bonds \\
\hline Dithymoquinone & -7.2 & 4 & Van der Waals, hydrogen bonds, \\
\hline Ritonavir & -6.4 & 5 & Van der Waals, hydrogen bonds \\
\hline Remdesivir & -6.3 & 6 & Van der Waals, hydrogen bonds \\
\hline Quinine & -6.2 & 7 & Van der Waals, hydrogen bonds \\
\hline Ferulic acid & -6.1 & 8 & Van der Waals, hydrogen bonds \\
\hline Syringin & -6.1 & 8 & Van der Waals, hydrogen bonds \\
\hline 6- gingerol & -6.1 & 8 & $\begin{array}{l}\text { Van der Waals, hydrogen bonds, carbon } \\
\text { hydrogen bond, alkyl and pi-alkyl interactions }\end{array}$ \\
\hline 6- paradol & -6.1 & 8 & Van der Waals, hydrogen bonds \\
\hline Hydroxychloroquine & -6.0 & 9 & $\begin{array}{l}\text { Van der Waals, hydrogen bonds, amide pi- } \\
\text { stacked and alkyl interactions }\end{array}$ \\
\hline Thymoquinone & -5.9 & 10 & Van der Waals, hydrogen bonds \\
\hline Thymohydroquinone & -5.8 & 11 & Van der Waals, hydrogen bonds \\
\hline Oleanolic acid & -5.8 & 11 & Van der Waals, hydrogen bonds \\
\hline Oseltamivir & -5.7 & 12 & Van der Waals, hydrogen bonds \\
\hline Lopinavir & -5.7 & 12 & $\begin{array}{l}\text { Van der Waals, hydrogen bonds, alkyl and pi- } \\
\text { alkyl interactions }\end{array}$ \\
\hline Gingerdione & -5.6 & 13 & Van der Waals, hydrogen bonds \\
\hline Thymol & -5.6 & 13 & Van der Waals, hydrogen bonds \\
\hline Protocatechuic acid & -5.5 & 14 & Van der Waals, hydrogen bonds \\
\hline Sinapic acid & -5.4 & 15 & Van der Waals, hydrogen bonds \\
\hline Umifenovir & -5.3 & 16 & Van der Waals, hydrogen bonds \\
\hline p- cymene & -5.3 & 16 & Van der Waals, hydrogen bonds \\
\hline Favipiravir & -5.2 & 17 & Van der Waals, hydrogen bonds \\
\hline Limonene & -5.2 & 17 & Van der Waals, hydrogen bonds \\
\hline Chloroquine & -4.6 & 18 & Van der Waals, hydrogen bonds \\
\hline$\alpha$ - pinene & -4.5 & 19 & Van der Waals, hydrogen bonds \\
\hline Citronellal & -4.5 & 19 & Van der Waals, hydrogen bonds \\
\hline$\beta$ - pinene & -4.2 & 20 & Van der Waals, hydrogen bonds \\
\hline Allicin & -3.7 & 21 & Van der Waals, hydrogen bonds \\
\hline
\end{tabular}

Molecules in bold are currently used in the therapeutic management of Covid-19

Table 3: Plants with Bioactive Compounds Showing Better Binding Affinity than Current Therapies to the SARSCoV-2 Molecular Target (7BV2.pdb)

\begin{tabular}{ll}
\hline Current Treatment & Plants having molecules with better binding affinity \\
\hline Remdesivir, Ritonavir and Quinine & Garcinia kola (Bitter kola); Viscum album (Mistletoe); \\
& Nigella sativa (Black seed) \\
Hydroxychloroquine, Lopinavir, Umifenovir, & Garcinia kola (Bitter kola); Viscum album (Mistletoe); \\
Favipiravir, Oseltamivir, and Chloroquine & Nigella sativa (Black seed); Aframomum melegueta \\
& (Alligator pepper) \\
\hline
\end{tabular}




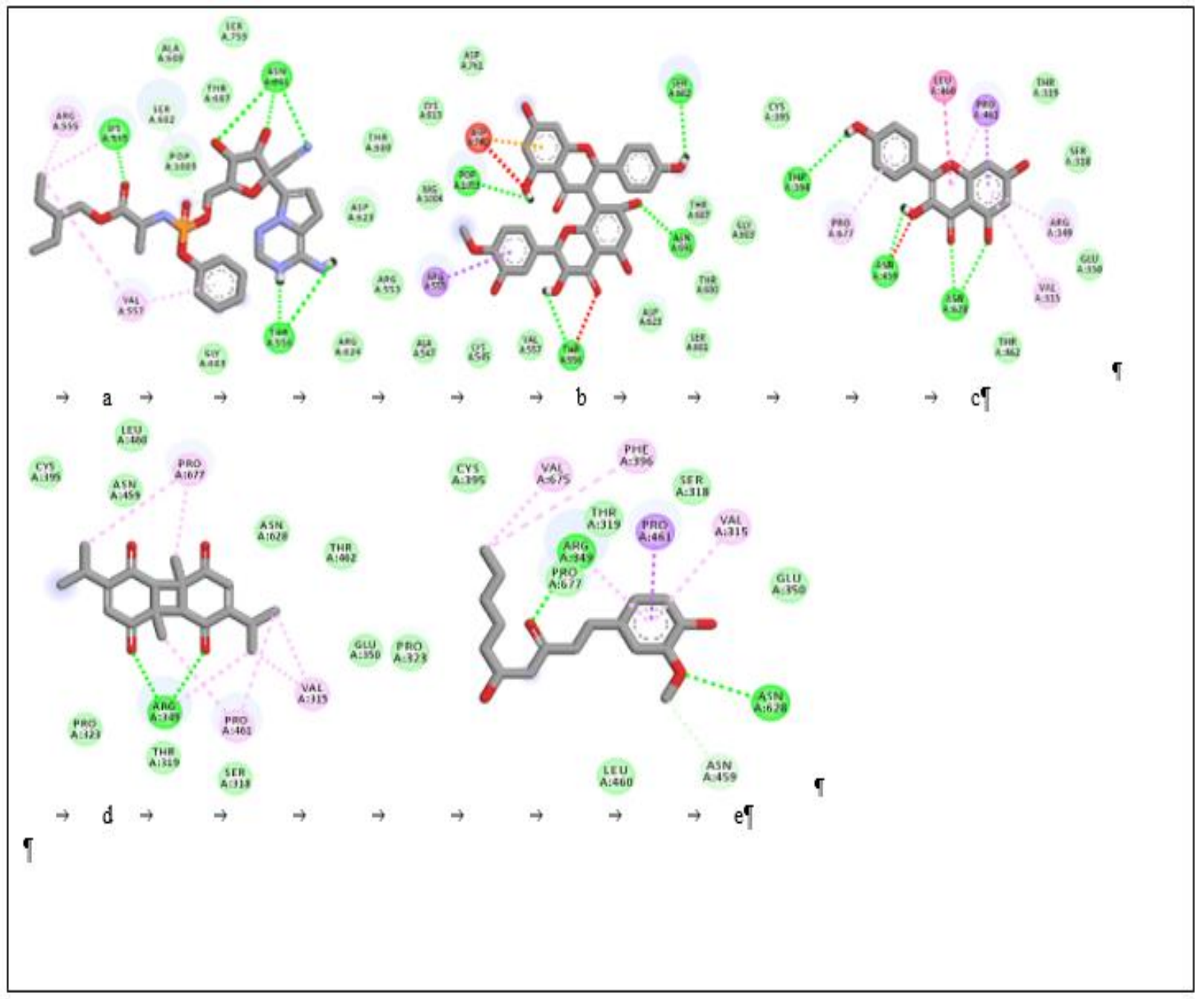

Figure 1: 2D binding interactions of Remdesivir (a), Kolaviron (b), kaempferol (c), Dithymoquinone (d) and 6gingerol (e) to the active site residues of SARS-CoV-2 (7BV2.pdb). Ligands are shown in stick forms while amino acid residues are shown in disc forms. Hydrogen bond interactions with amino acid main chain are indicated by green discontinuous lines, green coloured discs show van der Waal's interaction while purple discs show pi- sigma interactions. Discs and lines shown in red colour represent unfavourable bumps and interactions. 


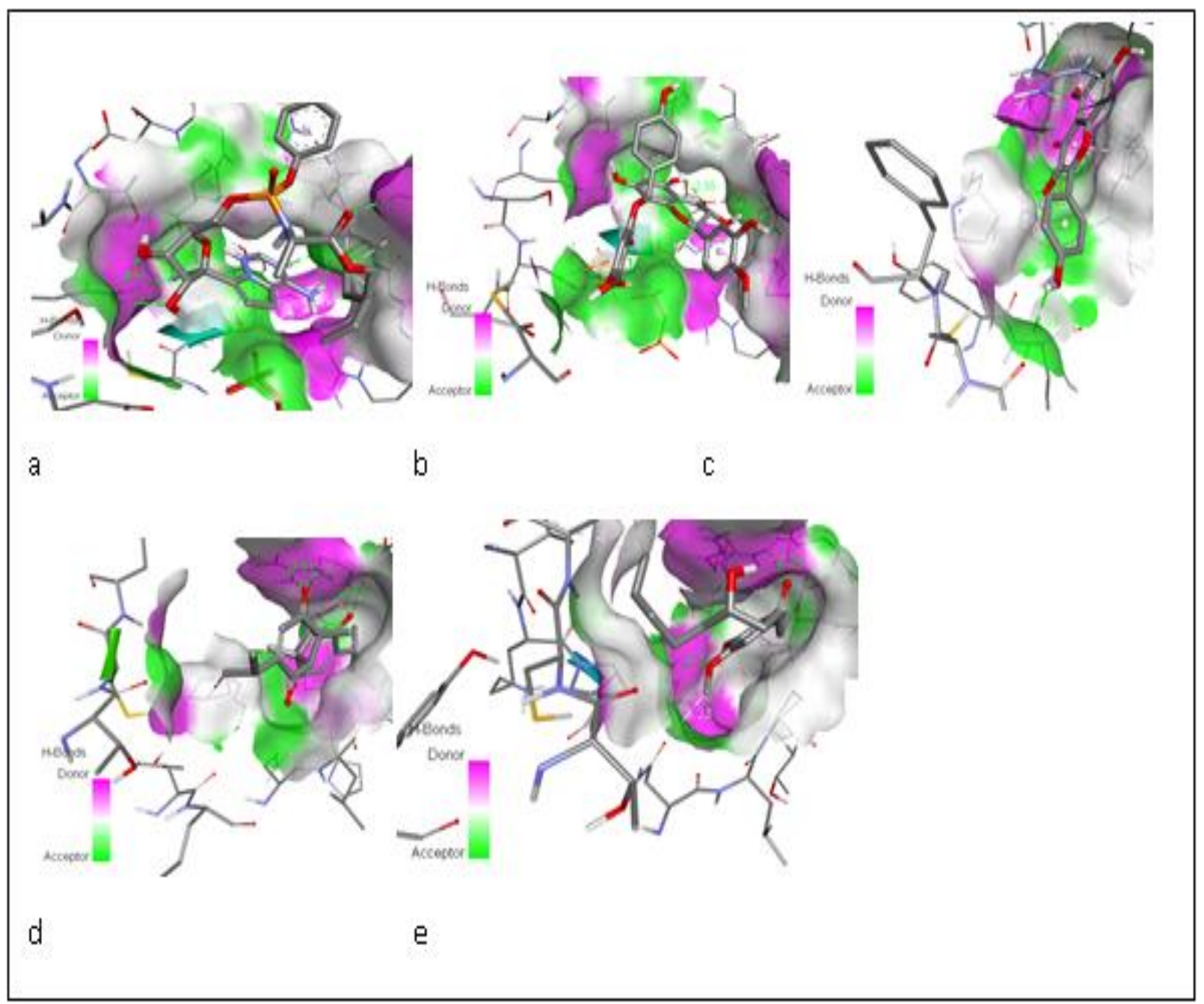

Figure 2: 3D view of binding conformation of Remdesivir (a), Kolaviron (b), kaempferol (c), Dithymoquinone (d) and 6- gingerol (e) to the active site residues of SARS-CoV-2 (7BV2.pdb) showing hydrogen bond length of between 2.11 to $2.63 \mathrm{~A}$.

A few unfavourable interactions were noticed (depicted in Figure 1a and Figure 1c) among some of the compounds with the target (7BV2.pdb) active residues. Threedimensional view of the binding in Figure $2 b$ is similar to that of Figure $2 \mathrm{a}$ as they are both seen to occupy similar active site pockets. This could be possibly be attributed to bulkiness of the compounds. Figure $2 \mathrm{c}-\mathrm{e}$ shows a conformation that suggests being buried in a much deeper pocket in the protein target. Studies [21] have shown the interactions of remdesivir with side chains of Lys 545 and Arg 555 which can also be observed in the interactions of kolaviron (Figure 1b). This is important as the residues Lys 545 and Arg 555 side chains are involved in stabilizing incoming nucleotide for correct positioning for catalysis. Phytochemicals of interest would be those that could inhibit the interaction of the primer strand RNA, which could in turn inhibit process of catalysis. In addition, kolaviron was also noted to have interacted with residues Asp 760 and Asp 761 which are responsible for coordination of magnesium ions at the catalytic centre of the protein.

As seen in Table 3, phytochemical compounds found in Garcinia kola, Viscum album and Nigella sativa had a better binding affinity for the target compared to remdesivir, 
ritonavir and quinine. Kolaviron showed the best binding energy of all the compounds docked (Table 1), and most of its hydrogen bond lengths are shorter in comparison with the other compounds (Figure 3). The shorter bond lengths observed could indicate a higher bond order which means the atoms are held more tightly together and therefore have a greater stability. Five bioactive compounds present in Viscum album (Mistletoe) were seen to have a better binding affinity than three current coronavirus therapy (Table 2). These results are in line with earlier reports affirming the antiviral activity of Viscum album (Mistletoe) [22]. When compared with the other therapeutic agents currently in use and included in this computational evaluation, all the medicinal plants in Vangag had some bioactive compound with a better binding affinity (Table 3). For example, 6- gingerol has been shown in a previous study to have high binding affinity to several COVID-19 viral target proteins [23]. Vangag also contains Gongronema latifolium which has been shown to have an appreciable amount of zinc and other vitamins [24] which is important in the management of COVID-19 [25].

In silico techniques have been shown to be capable of saving time on the often expensive exploratory animal and in vitro laboratory assays, and can quickly point out candidate formulations, plants or moieties, that might warrant further evaluation [26]. The computational simulations in this study have shown that Vangag herbal preparation contain phytochemical compounds that have favourable binding and inhibitory activities at the active site of SARS-CoV-2 protease. Vangag herbal preparation is therefore a promising formula and could be beneficial in the management of COVID-19.

\section{Acknowledgement.}

We acknowledge the University of Jos for the use of facilities.

\section{REFERENCES}

1. Zhang H, Liang F, Chen R. Ancient Records and Modern Research on the Mechanisms of Chinese Herbal Medicines in the Treatment of Diabetes Mellitus. Evid Based Complement Alternat Med 2015; http://dx.doi.org/10.1155/2015/747982

2. Gu S, Pei J. Innovating Chinese Herbal Medicine: From Traditional Health Practice to Scientific Drug Discovery. Front Pharmacol 2017;8:381. https://doi.org/10.3389/fphar.2017.00381

3. Ekor M. The growing use of herbal medicines: issues relating to adverse reactions and challenges in monitoring safety. Front Pharmacol 2014;4:177. https://doi.org/10.3389/fphar.2013.00177

4. Wu R, Wang L, Kuo HD, Shannar A, Peter R, Chou PJ, et al. An Update on Current Therapeutic Drugs Treating COVID-19. Curr Pharmacol Rep 2020;1-15. Advance online publication. https://doi.org/10.1007/s40495-020-00216-7

5. Yang Y, Islam MS, Wang J, Li Y, Chen X. Traditional Chinese Medicine in the Treatment of Patients Infected with 2019-New Coronavirus (SARSCoV-2): A Review and Perspective. Int J Biol Sci 2020;16(10): 1708 $-17$. https://doi.org/10.7150/ijbs.45538

6. Lin CQ. Traditional Chinese medicine is a resource for drug discovery against 2019 novel coronavirus (SARS-CoV-2). J Integr Med 2020;8(2):87- 8 . https://doi.org/10.1016/j.joim.2020.02.004

7. Zhang D, Wu KL, Zhang X, Deng SQ, Peng, B. In silico screening of Chinese herbal medicines with the potential to directly inhibit 2019 novel coronavirus. J Integr Med 2020;18(2):152-8. https://doi.org/10.1016/j.joim.2020.02.005

8. Ekins $\mathrm{S}$, Mestres $\mathrm{J}$, Testa $\mathrm{B}$. In silico pharmacology for drug discovery: methods for virtual ligand screening and profiling. $\mathrm{Br} \mathrm{J}$ Pharmacol 2007;152(1):9-20

https://doi.org/10.1038/sj.bjp.0707305

9. Tane P, Tatsimo SD, Ayimele GA, Connolly JD. Bioactive metabolites from Afromomum Species. In $11^{\text {th }}$ NAPRECA Symposium Book of Proceedings 2005;214:214-23.

10. El-Saber Batiha G, Magdy Beshbishy AG. Wasef, L.; Elewa, Y.H.A.; A. Al-Sagan, A.; Abd ElHack, M.E.; Taha, A.E.; M. Abd-Elhakim, Y.; Prasad Devkota, H. Chemical Constituents and 
Pharmacological Activities of Garlic (Allium sativum L.): A Review. Nutrients 2020, 12, 872

11. Mikaili P, Maadirad S, Moloudizargari M, Aghajanshakeri S, Sarahroodi S. (2013). Therapeutic uses and pharmacological properties of garlic, shallot, and their biologically active compounds. Iranian Journal of Basic Medical Sciences, 16(10), 1031-48.

12. Pietrzak W, Nowak R, Gawlik-Dziki U, Lemieszek MK, Rzeski W. (2017). LC-ESI-MS/MS Identification of Biologically Active Phenolic Compounds in Mistletoe Berry Extracts from Different Host Trees. Molecules (Basel, Switzerland), 22(4), 624. https://doi.org/10.3390/molecules22040624

13. Anchang KY, Garba M, Manjong FT, Tiagueu YT (2015). Potentials of nutritional therapy, phytopharmaceuticals and phytomedicine in the prevention and control of Ebola virus in Africa. American Journal of Clinical and Experimental Medicine 3(1-1) 1-6. https://doi.org/10.11648/j.ajcem.s.2015030101.11

14. Nickavar, B; Mojab, F., Javidnia, K; Amoli, M. A. (2003). Chemical composition of the fixed and volatile oils of Nigella sativa L. from Iran. J. Biosci., 58 (2003), 629-31.

15. Rajabian, A., Hosseinzadeh, H. (2020). Dermatological Effects of Nigella sativa and its Constituent, Thymoquinone: A Review. In: , Editor(s): V. R. Preedy, R. R. Watson (Eds). Nuts and Seeds in Health and Disease Prevention (Second Edition), Academic Press, 329-355.

16. Kim S, Chen J, Cheng T, Gindulyte A, He J, He S, et al. PubChem 2019 update: improved access to chemical data. Nucleic Acids Res 2019;47(D1), D1102-D1109. https://doi.org/10.1093/nar/gky1033

17. Dassault Systemes BIOVIA (2016) Discovery Studio Modeling Environment, Release 2017, San Diego: Dassault Systems.

18. Dallakyan, S, Olson, AJ. Small-Molecule Library Screening by Docking with PyRx. Methods Mol Biol 2015;1263:243-50. https://doi.org/10.1007/978-1-4939-2269-7_19
19. Trott O, Olson, AJ. AutoDock Vina: Improving the speed and accuracy of docking with a new scoring function, efficient optimization, and multithreading. J Comput Chem 2010;31(2):455-61. https://doi.org/10.1002/jcc.21334

20. DeLano WL. Pymol: An open-source molecular graphics tool.CCP4 Newsl. Protein Crystallogrphy 2002;40(1):82-92.

21. Yin W, Mao C, Luan X, Shen DD, Shen Q, Su, $\mathrm{H}$ et al (2020). Structural basis for inhibition of the RNA-dependent RNA polymerase from SARS-CoV-2 by remdesivir. Science eabc1560. https://doi.org/10.1126/science.abc1560

22. Karagöz A, Onay E, Arda N, Kuru A. Antiviral potency of mistletoe (Viscum album ssp. album) extracts against human parainfluenza virus type 2 in Vero cells. Phytother Res 2003;17(5):560-2. https://doi.org/10.1002/ptr.1163

23. Thirumalaisamy R, Murugan P, Srinivasan $P$, Arjunan S, Selvankumar T. Phytochemical 6-Gingerol - A promising Drug of choice for COVID-19. Int J Adv Sci Eng 2020;6(4):1482-9. https://doi.org/10.29294/IJASE.6.4.2020.1482-1489

24. Balogun ME, Besong EE, Obimma JN, Mbamalu OS, Djobissie SFA. Gongronema Latifolium: A Phytochemical, Nutritional and Pharmacological Review. Journal of Physiology and Pharmacology Advances, 2016;6(1), 811-24. https://doi.org/10.5455/jppa.1969123104000

25. te Velthuis AJW, van denWorm SH, Sims AC, Baric RS, Snijder EJ, van Hemert MJ et.al. $\mathrm{Zn}(2+)$ inhibits coronavirus and arterivirus RNA polymerase activity in vitro and zinc ionophores block the replication of these viruses in cell culture. PLoS Pathog 2010;6(11): e1001176. https://doi.org/10.1371/journal.ppat.1001176

26. Valerio Jr LG. Application of advanced In silico methods for predictive modelling and information integration. Expert Opin Drug Metab Toxicol 2012;8(4):395-8. https://doi.org/10.1517/17425255.2012.664636 